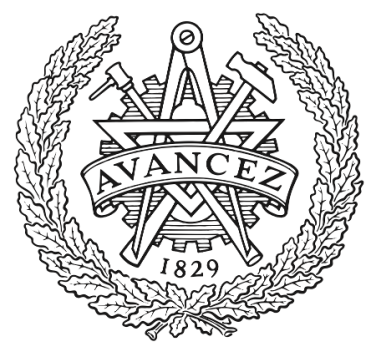

CHALMERS

UNIVERSITY OF TECHNOLOGY

\title{
11.5 bits/s/Hz PM-256QAM Comb-Based Superchannel Transmission by Combining Optical and Digital Pilots
}

Downloaded from: https://research.chalmers.se, 2023-04-26 15:32 UTC

Citation for the original published paper (version of record):

Mazur, M., Schröder, J., Lorences Riesgo, A. et al (2018). 11.5 bits/s/Hz PM-256QAM Comb-Based Superchannel Transmission by Combining Optical and Digital Pilots. 2018 OPTICAL FIBER COMMUNICATIONS CONFERENCE AND EXPOSITION (OFC). http://dx.doi.org/10.1364/OFC.2018.M1G.2

N.B. When citing this work, cite the original published paper. 


\title{
11.5 bits/s/Hz PM-256QAM Comb-Based Superchannel Transmission by Combining Optical and Digital Pilots
}

\author{
Mikael Mazur $^{(1)}$, Jochen Schröder ${ }^{(1)}$, Abel Lorences-Riesgo ${ }^{(1,2)}$, Tsuyoshi Yoshida ${ }^{(1,3)}$, Magnus Karlsson ${ }^{(1)}$, \\ Peter A. Andrekson ${ }^{(1)}$ \\ (1) Photonics Laboratory, Department of Microtechnology and Nanoscience, Chalmers University of Technology, Gothenburg, Sweden \\ (2) Now at IT-Instituto de Telecomunicações, 3810-193 Aveiro, Portugal \\ (3) Mitsubishi Electric Corporation, 5-1-1 Ofuna, Kamakura, Kanagawa, 247-8501 Japan \\ mikael.mazur@chalmers.se
}

\begin{abstract}
We demonstrate $44 \mathrm{~Tb} / \mathrm{s}$ transmission using three $50 \times 24$ Gbaud PM-256QAM comb-based superchannels. Each superchannel combines a single optical pilot tone with individual digital pilot symbols to minimize the total overhead, enabling record spectral-efficiency over the full C-band.

OCIS codes: (060.1660) Coherent communications; (070.1170) Analog optical signal processing
\end{abstract}

\section{Introduction}

In the last few years we have seen large improvements in fiber throughput thanks mainly to space division multiplexing (SDM), and using $>100$ spatial modes the aggregated throughput and spectral efficiency (SE) have reached beyond $10 \mathrm{~Pb} / \mathrm{s}$ and $1 \mathrm{kbit} / \mathrm{s} / \mathrm{Hz}$ respectively [1]. Full exploitation of system potential requires effective use of each individual fiber mode, and increasing the SE over a single fiber mode is of fundamental importance both for single-mode and SDM systems given the limited available bandwidth (BW). For single carrier signals, a record SE of $15.3 \mathrm{bits} / \mathrm{s} / \mathrm{Hz}$ was reported in [2]. The baudrate was limited to 3 Gbaud to cope with the limited effective number of bits in the transceiver and self-homodyne detection using a narrow-linewidth transmitter laser was required to avoid severe degradation due to laser phase noise. For wavelength division multiplexing (WDM) transmission, $14.2 \mathrm{bits} / \mathrm{s} / \mathrm{Hz}$ achievable information rate (AIR) was reported for $5 \times 16$ Gbaud signals modulated with probabilistically shaped 256 quadrature amplitude modulation (PS)-256QAM [3]. For transmission bandwidth exceeding $2 \mathrm{~nm}$ and approaching fully loaded systems, the SE record dates back to 2011 when 11 bits/s/Hz SE was achieved using orthogonal frequency division multiplexing [4]. The low baudrate mitigated digital-to-analog and analog-to-digital converter (DAC/ADC) limitations and digital pilot tones were used to avoid penalty arising from laser phase noise.

However, higher baudrates are needed to avoid unrealistic large channel counts. To cope with BW limitations, fiber impairment and carrier recovery of typically employed external cavity lasers (ECLs) with up to $100 \mathrm{kHz}$ linewidth, systems using high modulation formats typically employ powerful digital signal processing (DSP) based on digital pilots, which implies a loss in SE and pilot overheads $(\mathrm{OH})$ are reaching $10 \%$ for PM-256QAM [5]. The minimization of the total pilot $\mathrm{OH}$ is of critical importance to maximize throughput of each channel, as even a small additional $\mathrm{OH}$ for each channel translates to significant loss in total throughput when scaling to WDM or superchannel systems.

In this work, we investigate the combination of comb-based superchannels and shared optical pilot tones, proposed in [6], to improve the SE in high throughput systems. In contrast to previous work using a single pilot tone and blind DSP [6], we study comb-based superchannels using PM-256QAM and a combination of optical and digital pilots to minimize the total $\mathrm{OH}$. We use a standard ECL with $100 \mathrm{kHz}$ specified linewidth to seed our transmitter comb and transmission of a single pilot tone allows for locking the receiver comb seed laser to the transmitter. This removes the frequency offset and reduces the phase noise normally arising from differences between the transmitter and receiver lasers in intradyne detection, leading to relaxed requirements for the digital pilot symbol based carrier recovery. Using the proposed scheme, we demonstrate transmission of three 50 $\times 24$ Gbaud PM-256QAM superchannels over a single span of $40 \mathrm{~km}$ standard single-mode fiber (SSMF) with erbium doped fiber amplifier (EDFA)-only amplification. The resulting spectral efficiency of $11.5 \mathrm{bits} / \mathrm{s} / \mathrm{Hz}$ and corresponding AIR of $12 \mathrm{bits} / \mathrm{s} / \mathrm{Hz}$, achieved over the $31 \mathrm{~nm}$ bandwidth, is the highest reported SE and AIR for a signal bandwidth above $2 \mathrm{~nm}$. The results show the potential for comb-based superchannels combined with optical pilot tones to maximize the SE in dense WDM systems.

\section{Experimental setup}

The experimental setup is shown in Fig. 1. We followed a test-band/load-band approach similar to [1,6]. The $31 \mathrm{~nm}$ signal was generated from three $10.2 \mathrm{~nm}$ comb-based superchannels. Each superchannel consisted of 51 carriers spaced at $25 \mathrm{GHz}$ and generated from two electro-optic frequency combs seeded by one (comb containing the test-band) and two (load-combs) $100 \mathrm{kHz}$ linewidth ECLs respectively. The three superchannels were centered at 1539.8, 1550.1, $1560.6 \mathrm{~nm}$ and separated by $25 \mathrm{GHz}$ guard-bands. The test-comb was amplified and a wavelength selective switch (WSS) separated 5 neighboring lines from the comb to form the test-band. Even and odd lines were independently 


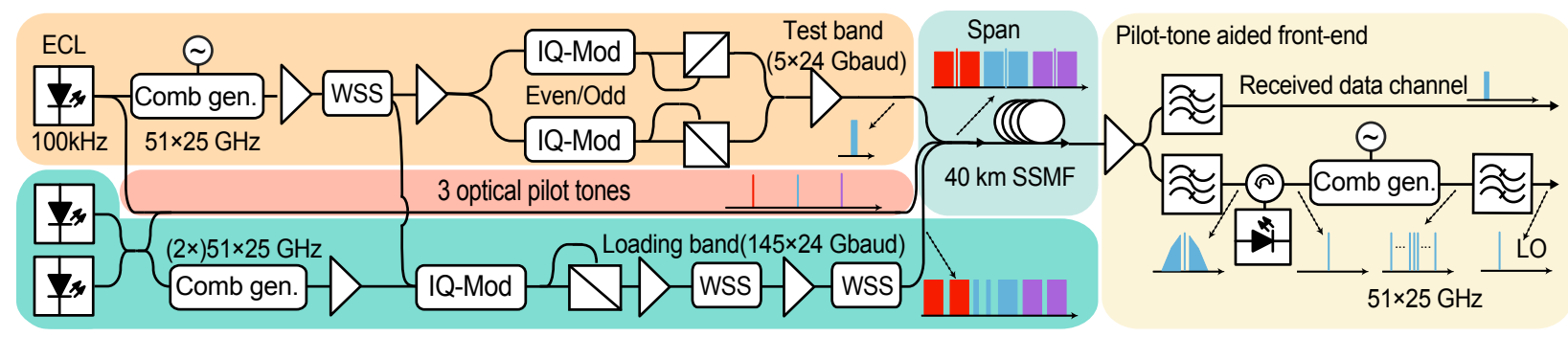

Fig. 1: Schematic of the PM-256QAM superchannel transmission setup.

modulated using two IQ-modulators, driven by four independent DACs operating at $60 \mathrm{GS} / \mathrm{s}$ to create a $24 \mathrm{Gbaud}$ 256QAM data signal shaped with a root-raised cosine filter (1\% roll-off). Linear pre-compensation of the DAC lowpass response and a 3-symbol memory look-up table based compensation scheme were used to mitigate transmitter distortions. Polarization multiplexing was emulated using the split-delay-combine method with $\sim 8 \mathrm{~ns}$ delay and the even and odd channels were individually amplified before recombination in an optical interleaver (OI). The remaining carriers from the test-comb were combined with the carriers from the loading-combs and modulated with PM256QAM data to form the loading-band. After amplification, an OI separated the loading channels into two arms and a delay of $\sim 250 \mathrm{~ns}$ was inserted in one arm before recombination in a second OI. The full loading band was then amplified and two cascaded WSSes were used to ensure spectral flatness and amplified spontaneous noise noise suppression at the location of the test-band and the optical pilot tones.

Taps from the lasers feeding the EO-combs were used to generate the shared optical pilot tones which were then combined with the modulated test and loading channels. The combined spectrum can be seen at the bottom of Fig. 3 , with the location of the optical pilots indicated by red lines. The three superchannels were transmitted over $40 \mathrm{~km}$ of SSMF with span loss of about $9 \mathrm{~dB}$ at a combined launch power of $14 \mathrm{dBm}$, which was the maximum available launch power and found to result in the best overall performance. Due to limitations in the available laser output power, the pilot tone launch power was about $6 \mathrm{~dB}$ lower than the data channels. The available launch power, and hence the distance, was limited by the available EDFA power $(\sim 22 \mathrm{dBm})$ and the insertion loss $(\sim 7 \mathrm{~dB})$ of the WSS together with the requirement of achieving a flat output spectrum.

The superchannel receiver using hybrid optical and digital carrier recovery is explained in Fig. 2. First, a shared analog carrier recovery was implemented using the optical pilot tone to lock the transmitter and receiver comb seed lasers. Following this, sparsely placed digital pilot symbols were used to compensate the residual phase variations on each individual wavelength channel within the superchannel. To recover the pilot tone we used optical filtering $(0.3 \mathrm{~nm}$ BW) followed by injection-locking of a standard distributed-feedback laser (free-running linewidth of $\sim 1 \mathrm{MHz}$ ) at an injection ratio of $-40 \mathrm{~dB}$. The slave laser output was fed to a separate receiver EO-comb generator driven using an independent RF-clock (not connected to the transmitter), to generate the local oscillator (LO) lines. Due to the nature of the injection locking process, the generated LO lines are phase locked to the transmitter comb and residual phase noise is mainly determined by the relative frequency offset ( $<50 \mathrm{kHz}$ over several days) and phase noise of the transmitter and receiver clocks. After bandpass filtering, the data channel and selected LO line were combined in a coherent optical receiver and sampled using a $50 \mathrm{GS} / \mathrm{s}$ scope with $23 \mathrm{GHz}$ analogue bandwidth.

Following resampling to 2 samples per symbol we used a pilot-based DSP to equalize the signal and remove residual carrier offset. The pilot frame structure is outlined in Fig. 2, and the total frame length was 104704 symbols (length determined by the memory of our arbitrary waveform generator). We used 1024 quadrature phase shift keying (QPSK) pilots in the beginning of the frame followed by the 256QAM payload. The initial pilot sequence was used for frame
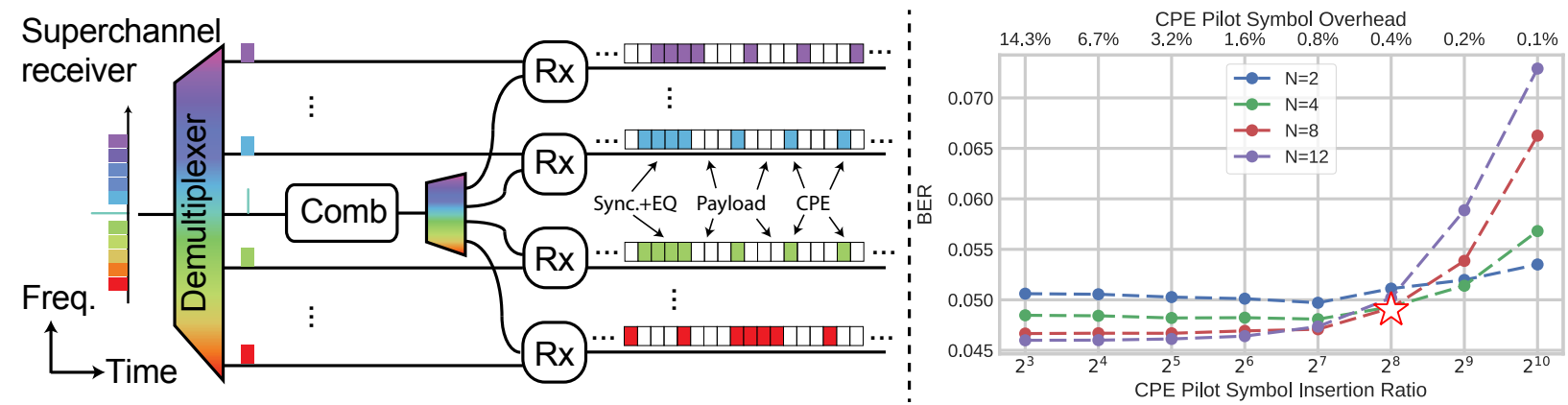

Fig. 2: (left) Principle sketch for the proposed scheme using a single pilot tone for locking the receiver comb seed laser and individual pilot symbols for tracking the residual phase variations. (right) Required digital pilot symbol insertion ratio and overhead. 


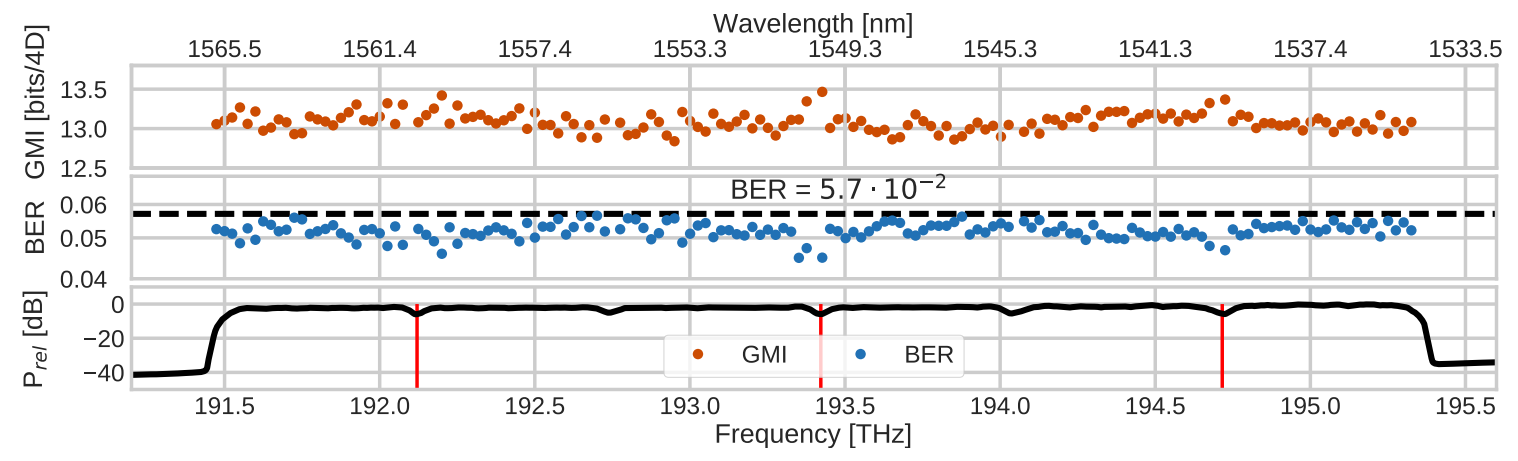

Fig. 3: Bit error rate (BER), generalized mutual information (GMI) after transmission together with the transmitted spectrum. Vertical red lines indicates the frequency of the optical pilot tones and the center frequency for each superchannel.

synchronization and to converge a $2 \times 2$ complex equalizer with 45-T/2-spaced taps used for equalization of the complete frame. No equalizer pre-convergence using additional pilots or training using the payload was used. Within the payload, additional QPSK pilot symbols were inserted for pilot-based phase tracking (CPE) to cancel out any residual phase variations. Finally, performance was evaluated using generalized mutual information (GMI) and bit error rate (BER), both calculated using the frame payload (about $3 \cdot 10^{6}$ bits) and averaged over 3 separate measurements.

\section{Results}

To minimize the combined overhead of the optical and digital pilots, we studied the required digital pilot symbol insertion ratio for phase tracking using self-homodyne detection in a single-carrier back-to-back scenario. We swept both the ratio and the block length $(\mathrm{N})$ of the moving-average filter used to average out noise on the pilot symbols as shown in Fig. 2. We found a 1/256 minimum pilot symbol insertion ratio (corresponding to an $\mathrm{OH}$ of $0.4 \%$ ) and blocksize of 8 was needed to minimize the $\mathrm{OH}$ without a significant BER penalty (star in Fig. 2). With the $2 \%$ overhead for transmitting the optical pilot tone, the total overhead for phase tracking was $2.4 \%$. Apart from the pilot symbols for phase tracking, we allocated a $1 \%$ overhead for the pilot sequence used for equalization and synchronization. This $\mathrm{OH}$ was sufficient in the case of pilot-tone aided transmission but resulted in more than $10 \%$ BER in the case of intradyne detection using an additional free-running ECL as LO. This was caused by imperfect equalizer convergence due to inaccurate frequency offset estimation. The estimation was done using the same pilots as for the equalizer convergence and an about five times longer pilot sequence was needed to overcome this issue when not using the pilot tone.

Finally, we evaluated the performance of the proposed scheme for broad-band high-SE transmission. The BER and GMI for each of the 150 lines after transmission are shown in Fig. 3. The BER for all channels is below 5.7\%, which corresponds to our BER threshold assuming $28 \%$ overhead SD-FEC [7]. Taking into account only coding overhead, the channel SE would be $12.5 \mathrm{bits} / \mathrm{s} / \mathrm{Hz}$. GMI measurements show that this SE is achievable since minimum and average GMIs are 12.9 and $13.1 \mathrm{bits} / \mathrm{s} / \mathrm{Hz}$. The final SE was $11.5 \mathrm{bits} / \mathrm{s} / \mathrm{Hz}$ accounting for a reduction due to: the $3.4 \%$ for optical and digital pilots, $4 \%$ guard bands between data channels and $1.3 \%$ guard bands between superchannels and the $28 \%$ coding overhead. The AIR ${ }_{W D M}$ [3] calculated from the GMI and including pilot overheads was $12 \mathrm{bits} / \mathrm{s} / \mathrm{Hz}$. The resulting total throughput for the two cases was $44.4 \mathrm{~Tb} / \mathrm{s}$ and $46.5 \mathrm{~Tb} / \mathrm{s}$ respectively.

\section{Conclusions}

We have proposed and experimentally studied comb-based superchannels for high spectral-efficiency transmission. The spectral coherence is exploited for effective sharing of a pilot tone which combined with individual pilot symbol based DSP for each sub-channel, enables signal recovery with minimized overhead. Using this, we have demonstrated simultaneous transmission of $3 \times 50 \times 300 \mathrm{~Gb} / \mathrm{s} 24-\mathrm{Gbaud} \mathrm{PM}-256 \mathrm{QAM}$ superchannels. Our reported spectral efficiency of $11.5 \mathrm{bits} / \mathrm{s} / \mathrm{Hz}$ and $\mathrm{AIR}_{W D M}$ of $12 \mathrm{bits} / \mathrm{s} / \mathrm{Hz}$ (taking into account all pilot overheads) is, to the best of our knowledge, the highest SE demonstrated for a transmission bandwidth exceeding $2 \mathrm{~nm}$.

\section{Acknowledgements}

This work was supported by the Swedish Research Council (grant 2014-06138).

\section{References}

[1] D. Soma et al., "10.16 Peta-bit/s Dense SDM/WDM Transmission over Low-DMD 6-Mode 19-Core Fibre...,” Proc. ECOC Th.PDP.A.1, (2017).

[2] S. Beppu et al., "2048 QAM (66 Gbit/s) single-carrier coherent optical transmission over 150 km...," Opt. Express, 23(4), pp. 4960-4969 (2015).

[3] S. Chandrasekhar et al., "High-spectral-efficiency transmission of PDM 256-QAM with Parallel Probabilistic...," Proc. ECOC, Th.3.C.1, (2016).

[4] D. Qian et al., "101.7-Tb/s (370x294-Gb/s) PDM-128QAM-OFDM transmission over 3×55-km SSMF...", Proc. OFC, PDPB5, (2011).

[5] F. Da Ros et al., "Characterization and optimizatiopn of a high-efficiency AlGaAs-On-Isulator-based...," JLT, 35(17), pp. 3750-3757, (2017).

[6] M. Mazur et al., "10.3 bits/s/Hz Spectral Efficiency 54x24 Gbaud PM-128QAM Comb-Based Superchannel...," Proc. ECOC, M.1.F.5, (2017).

[7] F. Buchali et al., "Implementation of 64QAM at 42.66 GBaud using 1.5 samples per symbol DAC...," Proc. OFC, M.2.A.1, (2014). 